\title{
Combined Use of Duloxetine and Olanzapine in the Treatment of Urologic Chronic Pelvic Pain Syndromes Refractory to Conventional Treatment: A Case Report
}

\author{
Bo Bi ${ }^{1}$, Liping Shan ${ }^{2}$, Die Zhou ${ }^{1}$ \\ ${ }^{1}$ Department of Psychiatry, First Affiliated Hospital, China Medical University, ${ }^{2}$ Department of Urology, Shengjing Hospital of China Medical \\ University, Shenyang, PR China

\begin{abstract}
Patients with urologic chronic pelvic pain syndromes (UCPPS) report interstitial cystitis/bladder pain syndrome and/or chronic prostatitis/chronic pelvic pain syndrome. The pathogenesis of these syndromes remains unclear and there is currently no standard treatment. UCPPS is, therefore, often misdiagnosed and its management is complex. The present case report involves a 62-year-old male patient with UCPPS whose main presentation is painful bladder filling and painful urgency refractory to conventional treatment with medication, which was successfully treated with the combined use of duloxetine and olanzapine. The combined use of duloxetine and olanzapine may become a new therapeutic option in the management of UCPPS.
\end{abstract}

KEY WORDS: Bladder pain; Painful urinary urgency; Duloxetine; Olanzapine; Anxiety.

\section{INTRODUCTION}

Chronic prostatitis/chronic pelvic pain syndrome (CP/ CPPS) is characterized by chronic pain in the perineum, tip of the penis, suprapubic region, or scrotum which is often worsened with voiding or ejaculation. ${ }^{1)}$ Interstitial cystitis/painful bladder syndrome (IC/BPS) indicates the presence of pain referable to the suprapubic region, and is often accompanied by increased urinary frequency, persistent urgency to void, or nocturia, which are described under the umbrella term of urologic chronic pelvic pain syndromes (UCPPS). ${ }^{2)}$ In patients with UCCPS, examination of the bladder and prostate shows normal findings. Various treatment options have been proposed for UCPPS; however, there is currently no optimal approach. The use of antidepressant drugs for the management of chronic pelvic pain has been reported in the past. van

Received: October 12, 2016/Revised: January 21, 2017 Accepted: January 23, 2017

Address for correspondence: $\mathrm{Bo} \mathrm{Bi}, \mathrm{MD}, \mathrm{PhD}$

Department of Psychiatry, First Affiliated Hospital, China

Medical University, 155 North Nanjing Street, Heping District

Shenyang, Liaoning 110001, PR China

Tel: +86-13889336624, Fax: +86-2483282184

E-mail: skiffinbb@163.com
Ophoven and Hertle $\mathrm{e}^{3)}$ conducted a prospective case series in order to evaluate the efficacy and tolerability of duloxetine for interstitial cystitis, and Giannantoni et al. ${ }^{4)}$ evaluated the efficacy and safety of duloxetine hydrochloride in the treatment of patients affected by CP/CPPS. The conclusion is controversial.

Duloxetine, a dual reuptake inhibitor of serotonin and norepinephrine, is approved for the treatment of major depressive disorder, generalized anxiety disorder, diabetic peripheral neuropathic pain, fibromyalgia, and chronic musculoskeletal pain in the United States. ${ }^{5)}$ In Europe, duloxetine is also indicated for the treatment of stress urinary incontinence in women. ${ }^{6,7)}$ Although some studies have suggested that duloxetine may be effective in treating overactive bladder, ${ }^{8-10)}$ its use in this condition is still not indicated. ${ }^{11)}$ One study has aslo reported that the addition of duloxetine hydrochloride to a-blocker medication and a saw palmetto extract was superior in relieving pain and improving psychological status compared with a conventional treatment including the two drugs alone in patients affected by $\mathrm{CP} / \mathrm{CPPS}$. $^{4)}$ But very few studies have been performed in which antidepressant medication combined with antipsychotics have been used to treat patients affected by UCPPS. The present case report

(c) This is an Open-Access article distributed under the terms of the Creative Commons Attribution Non-Commercial License (http://creativecommons.org/licenses/by-nc/4.0) which permits unrestricted non-commercial use, distribution, and reproduction in any medium, provided the original work is properly cited. 
concerns a male patient diagnosed with UCPPS that was refractory to conventional treatment, but was successfully treated with duloxetine combined with low dose of olanzapine. Written informed consent to publish this case was obtained from the patient.

\section{CASE}

A 62-year-old male patient, a retired worker, presented with urgency and frequency for more than 20 years and a painful bladder filling and painful urinary urgency symptoms for five years, reported that his pain is worse with bladder filling and the pain can relieve after micturition. The patient denied urinary tract infection and bladder trauma history and complained of a five-year history of urologic chronic pelvic syndromes, including painful bladder filling and painful urgency. The filling bladder pain is severe with intervals of one to two hours and he had to urinate every one to two hours during the night. Each day when sleeping, it was necessary for him to wake up four or five times a night.

Before the patient was referred to our psychiatric department, he was admitted in our urological department and department of pain. Initial physical examination, urinalysis, X-rays and computed tomography scan of the lower abdomen yielded no abnormal findings. The ultrasonography, uroflowmetry, and cystoscopy were also performed in the patient and the results were also normal.

The patient responded poorly to oral gabapentin 100 $\mathrm{mg}$ three times/day and solifenacin $5 \mathrm{mg} /$ day. Under the pain specialist's care, he was prescribed nonsteroid anti-inflammatory drugs (ibuprofen $100 \mathrm{mg} /$ day) for two weeks, tylox for two weeks, with minimal benefit. However his painful urgency symptoms remained unimproved, leading him to worry that the symptoms were not treatable. He could not sleep at night because of the bladder pain and his pain makes concentration difficult.

A psychiatric consultation was requested because of an anxiety mood for nearly six months, related to the unremitting pain. He was then suggested to our psychiatric department and was diagnosed the generalized anxiety disorder with the symptoms of painful bladder filling and urgency of urination. He was then prescribed duloxetine (30 mg/day) and clonazepam (1-2 mg/day) and the dosage of duloxetine was escalated to $60 \mathrm{mg} /$ day after two weeks of titration and then $120 \mathrm{mg} /$ day after eight weeks.
Furthermore, we gradually reduced the dose of clonazepam to $0.5 \mathrm{mg}$ weekly, starting at admission day until its final discontinuation within two weeks. Eight weeks after duloxetine initiation, he described his urgency and bladder pain as $50 \%$ better. While he was taking duloxetine (120 mg/day), his anxiety symptoms including worry and agitation also improved along with his alleviation of painful bladder pain symptoms and urinary urgency and frequency over the next three months. Psychometric testing revealed a Hamilton anxiety score of 10 and Hamilton depression score of 8 .

He reported experiencing almost no change in his symptom of painful bladder filling and painful urgency after five months. The patient was then started on olanzapine $2.5 \mathrm{mg}$ at bedtime for insomnia. Within days of continuing duloxetine combined olanzapine, the patient reported decreased filling pain and frequency. His urination frequency decreased to five or fewer times a day and the complaints of urinary filling pain did not recur throughout treatment with duloxetine combined with olanzapine (2.5 $\mathrm{mg} /$ day). About three months later, he no longer had nocturnal, and his micturition frequency had decreased to every five hours during the night, allowing him to sleep well. The patient continued to receive treatment for the following four months. At one year of treatment, he no longer had trouble with daily living or with taking the bus.

\section{DISCUSSION}

To the best of our knowledge, this is the first study reporting a case of UCPPS with the symptoms of painful bladder filling and urinary urgency and anxiety which was successfully treated with duloxetine combined with olanzapine. This case demonstrated that the addition of low dose of olanzapine to duloxetine hydrochloride was superior in relieving pain and improving psychological status. In this case, there is little response when the painful bladder filling and painful urgency treatment begins with gabapentin and solifenacin and then the pain management with nonsteroid anti-inflammatory drugs, but prominent improvement after duloxetine administration.

UCPPS is primarily characterized by chronic and often debilitating pain in the pelvic region and/or genitalia and typically a spectrum of defects in bladder and lower urinary tract function. ${ }^{12)}$ The etiology of the syndrome is still poorly understood, and it is not clear whether UCPPS is a 
disease of the prostate gland or a voiding dysfunction, a myofascial pain syndrome, or a functional somatic syndrome. Recently, symptoms of UCPPS have been considered as the result of an interaction among psychological factors and dysfunction in the immune, neurologic, and endocrine systems. ${ }^{13)}$ The most frequent therapies for UCPPS include single or sequential treatments or multimodal approaches with antibacterial, analgesics, and anti-inflammatory drugs, a-blockers, and pelvic floor rehabilitation to treat dysfunctional voiding, but the treatment is not always effective. ${ }^{14)}$

Duloxetine belongs to serotonin-norepinephrine reuptake inhibitors and is usually used for the treatment of major depressive disorder, generalized anxiety, and fibromyalgia. It is currently approved by the US Food and Drug Administration for the management of diabetic peripheral neuropathic pain. ${ }^{15)}$ Duloxetine is also approved in Europe for the treatment of stress urinary incontinence and has also exhibited efficacy in clinical trials in treating overactive bladder. ${ }^{9,16,17)}$ At present, only sparse experiences are available regarding antidepressant treatment in patients with UCPPS. A previous observational study demonstrated that the use of duloxetine promised amelioration of interstitial cystitis symptoms in women, but did not result in significant improvement of symptoms. ${ }^{3)}$ The previous review also concluded that the use of antidepressants for the management of chronic urological pelvic pain was not adequately supported by methodologically sound randomized clinical trials. ${ }^{3)}$

The mechanism of action of duloxetine in decreasing the symptoms of UCPPS is most likely related to its dual inhibition of serotonin and norepinephrine reuptake at spinal and/or supraspinal levels. Duloxetine is well able to counterbalance voiding dysfunctions and urinary incontinence, acting on the Onuf's nucleus in the sacral spinal cord. ${ }^{18)}$ Furthermore, results of an animal study suggested that at high doses $(1-3 \mathrm{mg} / \mathrm{kg})$ duloxetine significantly increased bladder capacity. ${ }^{19)}$ Duloxetine's ability to increase bladder capacity may be an important contributor to the improvement of urgency and frequency in our case. The inhibition of 5-hydroxytryptamine (5-HT) and noradrenaline (NA) reuptake initiates central inhibitory mechanisms, resulting in suppression of detrusor hyperactivity and putatively resulting in suppression of urgency/frequency complaints in the patient with UCPPS. ${ }^{3)}$

It is found from this case that the symptoms of painful filling and painful urgency treated with duloxetine did decrease significantly after combing with low-dose olanzapine. So far, the mechanism by which second-generation antipsychotics may have additive or synergistic effects on UCPPS when added to serotonin-noradrenaline reuptake inhibitors remains unclear. The 5-HT and norepinephrine dysfunction from the brainstem to spinal cord is considered one of central mechanisms in the development of UCPPS. ${ }^{20)}$ It is hypothesized that inhibition of 5-HT and NA reuptake initiates central inhibitory mechanisms, resulting in suppression of detrusor hyperactivity and putatively resulting in suppression of urgency/frequency and pain complaints in the patient with UCPPS. ${ }^{3)}$ Duloxetine is a potent inhibitor of neuronal serotonin and norepinephrine reuptake and a less potent inhibitor of dopamine reuptake, metabolized by cytochromes CYP1A2 and CYP2D6. Olanzapine has affinity to various receptors, such as dopaminergic receptors (D1, D2, D3, and D4), serotonergic receptors (5-HT2A, 5-HT2C, 5-HT3, and 5-HT6), muscarinic receptors (M1, $M 2, M 3$, and $M 4)$, histaminergic receptor $(\mathrm{H} 1)$, and adrenergic receptor $\alpha 1{ }^{21)}$ The major metabolic pathways of olanzapine include direct $\mathrm{N}$-glucuronidation, mediated by UGT1A4, and $\mathrm{N}$-demethylation, mediated by CYP1A2 ${ }^{22)}$ Synaptic transmission in all pelvic and bladder ganglia is mediated by acetylcholine acting on postjunctional muscarinic receptors and M3 muscarinic receptor subtype is the principal receptor involved in excitatory transmission. ${ }^{23,24)}$ Muscarinic agonists also release a substance called urothelium-derived inhibitory factor that decreases the force of detrusor muscle contraction. ${ }^{25)}$ The additive effect of olanzapine is probably due to anticholinergic effect by atypical antipsychotics. Several clinical reports have documented a beneficial effect of adding atypical antipsychotic drugs to ongoing treatments with antidepressants in ameliorating chronic pain. ${ }^{26)}$ In this case, the augmentation of duloxetine therapy with low dose of olanzapine, the possibility of a pharmacokinetic interaction and pharmacodynamic drug interaction should be taken into account. The inclusion of duloxetine hydrochloride $(120 \mathrm{mg} /$ day $)$ with low dose of olanzapine $(2.5 \mathrm{mg} /$ day $)$ allowed a better result in controlling clinical symptoms in patient affected by UCPPS. 


\section{REFERENCES}

1. Riegel B, Bruenahl CA, Ahyai S, Bingel U, Fisch M, Löwe B. Assessing psychological factors, social aspects and psychiatric co-morbidity associated with Chronic Prostatitis/Chronic Pelvic Pain Syndrome (CP/CPPS) in men--a systematic review. J Psychosom Res 2014;77:333-350.

2. Forrest JB, Schmidt S. Interstitial cystitis, chronic nonbacterial prostatitis and chronic pelvic pain syndrome in men: a common and frequently identical clinical entity. J Urol 2004;172: 2561-2562.

3. van Ophoven A, Hertle L. The dual serotonin and noradrenaline reuptake inhibitor duloxetine for the treatment of interstitial cystitis: results of an observational study. I Urol 2007; 177:552-555.

4. Giannantoni A, Porena M, Gubbiotti M, Maddonni S, Di Stasi $\mathrm{SM}$. The efficacy and safety of duloxetine in a multidrug regimen for chronic prostatitis/chronic pelvic pain syndrome. Urology 2014;83:400-405.

5. Eli Lilly and Cmpany. Highlights of prescribing information. Indianapolis:Eli Lilly and Cmpany;2012.

6. Millard RJ, Moore K, Rencken R, Yalcin I, Bump RC; Duloxetine UI Study Group. Duloxetine vs placebo in the treatment of stress urinary incontinence: a four-continent randomized clinical trial. BJU Int 2004;93:311-318.

7. Guay DR. Duloxetine for management of stress urinary incontinence. Am J Geriatr Pharmacother 2005;3:25-38.

8. Xia D, Wang P, Chen J, Wang S, Jiang H. Fluoxetine ameliorates symptoms of refractory chronic prostatitis/chronic pelvic pain syndrome. Chin Med J (Engl) 2011;124:2158-2161.

9. Steers WD, Herschorn S, Kreder KJ, Moore K, Strohbehn K, Yalcin I, et al; Duloxetine OAB Study Group. Duloxetine compared with placebo for treating women with symptoms of overactive bladder. BJU Int 2007;100:337-345.

10. Wang SM, Lee HK, Kweon YS, Lee CT, Lee KU. Overactive bladder successfully treated with duloxetine in a female adolescent. Clin Psychopharmacol Neurosci 2015:13:212214.

11. Di Rezze S, Frasca V, Inghilleri M, Durastanti V, Cortese A, Giacomelli $\mathrm{E}$, et al. Duloxetine for the treatment of overactive bladder syndrome in multiple sclerosis: a pilot study. Clin Neuropharmacol 2012;35:231-234.

12. Clemens JQ, Mullins C, Kusek JW, Kirkali Z, Mayer EA, Rodríguez LV, et al; MAPP Research Network Study Group. The MAPP research network: a novel study of urologic chronic pelvic pain syndromes. BMC Urol 2014;14:57.
13. Pontari MA, Ruggieri MR. Mechanisms in prostatitis/chronic pelvic pain syndrome. J Urol 2008;179(5 Suppl):S61-S67.

14. Seth A, Teichman JM. What's new in the diagnosis and management of painful bladder syndrome/interstitial cystitis? Curr Urol Rep 2008;9:349-357.

15. Zilliox L, Russell JW. Maintaining efficacy in the treatment of diabetic peripheral neuropathic pain: role of duloxetine. Diabetes Metab Syndr Obes 2010;3:7-17.

16. Mariappan P, Alhasso A, Ballantyne Z, Grant A, N'Dow J. Duloxetine, a serotonin and noradrenaline reuptake inhibitor (SNRI) for the treatment of stress urinary incontinence: a systematic review. Eur Urol 2007;51:67-74.

17. Schwen Z, Matsuta Y, Shen B, Wang J, Roppolo JR, de Groat $\mathrm{WC}$, et al. Inhibition of bladder overactivity by duloxetine in combination with foot stimulation or WAY-00635 treatment in cats. Am J Physiol Renal Physiol 2013;305:F1663-F1668.

18. Jost W, Marsalek P. Duloxetine: mechanism of action at the lower urinary tract and Onuf's nucleus. Clin Auton Res 2004; 14:220-227.

19. Reese J, Xiao Z, Schwen Z, Matsuta Y, Shen B, Wang J, et al. Effects of duloxetine and WAY100635 on pudendal inhibition of bladder overactivity in cats. J Pharmacol Exp Ther 2014; 349:402-407.

20. de Groat WC, Griffiths D, Yoshimura N. Neural control of the lower urinary tract. Compr Physiol 2015;5:327-396.

21. Callaghan JT, Bergstrom RF, Ptak LR, Beasley CM. Olanzapine. Pharmacokinetic and pharmacodynamic profile. Clin Pharmacokinet 1999;37:177-193.

22. Spina $\mathrm{E}$, de Leon J. Metabolic drug interactions with newer antipsychotics: a comparative review. Basic Clin Pharmacol Toxicol 2007; 100:4-22.

23. Andersson KE, Arner A. Urinary bladder contraction and relaxation: physiology and pathophysiology. Physiol Rev 2004;84:935-986.

24. Matsui M, Motomura D, Fujikawa T, Jiang J, Takahashi S, Manabe $\mathrm{T}$, et al. Mice lacking M2 and M3 muscarinic acetylcholine receptors are devoid of cholinergic smooth muscle contractions but still viable. I Neurosci 2002;22:1062710632.

25. Kumar V, Cross RL, Chess-Williams R, Chapple CR. Recent advances in basic science for overactive bladder. Curr Opin Urol 2005; 15:222-226.

26. Khouzam HR. Psychopharmacology of chronic pain: a focus on antidepressants and atypical antipsychotics. Postgrad Med 2016; 128:323-330. 